\title{
OCORRÊNCIA DE Eimeria spp. EM REBANHOS BUBALINOS DE APTIDÃO \\ LEITEIRA NO SUL DO BRASIL
}

\author{
MOTTA, Sara Patron ${ }^{1}$; \\ MARTINS, Natália Soares ${ }^{1}$; \\ SANTOS, Carolina Caetano ${ }^{1}$; \\ MOREIRA, Andrios da Silva ${ }^{1}$; \\ DAMÉ, Maria Cecília Florisbal ${ }^{2}$; \\ FARIAS, Nara Amélia da Rosa ${ }^{3}$; \\ RUAS, Jerônimo Lopes ${ }^{4}$.
}

${ }^{1}$ Doutorando, Programa de Pós-Graduação em Microbiologia e Parasitologia, UFPEL; ${ }^{2}$ Médica Veterinária, Doutora, EMBRAPA Clima Temperado; ${ }^{3}$ Professora, Doutora, Departamento de Microbiologia e Parasitologia, UFPEL; ${ }^{4}$ Médico Veterinário, Doutor, Faculdade de Veterinária, UFPEL.

\section{RESUMO}

A criação de bubalinos tem se mostrado uma importante atividade no Brasil, porém infecções por parasitos gastrintestinais representam um ponto limitante para essa espécie. O coccídio do gênero Eimeria é umas das causas primárias de doenças parasitárias de ocorrência mundial, resultando em perdas econômicas e desenvolvimento de sinais clínicos como diarreia, refletindo diretamente na diminuição da produção. Diante desses fatos, o presente estudo teve como objetivo relatar a ocorrência de Eimeria spp. em bubalinos de aptidão leiteira da microrregião de Camaquã e de Cachoeira do Sul (Rio Grande do Sul, Brasil). Foram coletadas 539 amostras de fezes, diretamente da ampola retal. Os bubalinos foram divididos em duas categorias: jovens (até um ano de idade) e adultos (acima de um ano de idade). As amostras foram processadas pela técnica de Gordon e Whitlock modificada, a qual foi utilizada para obter a contagem de oocistos de Eimeria spp. O resultado foi expresso em oocistos por grama de fezes (OoPG). Constatou-se que $41,2 \%$ dos bubalinos jovens e $16,9 \%$ dos adultos foram positivos para Eimeria spp. Este estudo demonstrou a ocorrência de Eimeria spp. parasitando bubalinos nas microrregiões de Camaquã e Cachoeira do Sul, acometendo principalmente animais jovens.

Palavras-chave: Parasitos. Protozoário. Exame coprológico. Bubalus bubalis. 
A bubalinocultura tem se mostrado uma importante atividade para elevação da renda média na atividade agropastoril, auxiliando na permanência do homem no campo (BERNARDES, 2007). No Brasil existem 950.173 cabeças de búfalos e no estado do Rio Grande do Sul, a população atinge 45.710 animais. No Censo Agropecuário de 2006 havia 885.119 cabeças, um incremento de 7,4\% em 11 anos, enquanto no mesmo período houve uma redução de $2 \%$ no efetivo de bovinos (IBGE, 2006, 2017).

A intensificação dos sistemas de produção com consequente aumento da lotação animal nos estabelecimentos pecuários favoreceram a disseminação de doenças infecciosas e parasitárias nos rebanhos. Os bubalinos, mesmo com sua reconhecida resistência, sofrem com esses novos desafios sanitários (BETHENCOURT et al., 2013). A ação de parasitos gastrintestinais é considerada um ponto limitante para a produção de ruminantes em geral (VILELA et al., 2017).

Protozoários coccídios são umas das causas primárias de doenças parasitárias de ocorrência mundial, resultando em perdas econômicas e desenvolvimento de sinais clínicos como diarreia, o que reflete diretamente na diminuição da produção (DANTAS et al., 2015).

Eimeria spp. é um coccídio de multiplicação intracelular, que acomete as células intestinais do hospedeiro. Os oocistos são eliminados nas fezes, após o processo de esporulação no ambiente tornam-se infectantes e aptos a parasitar outros animais do rebanho. Conforme o nível de ingestão de oocistos, podem desenvolver diferentes graus de morbidade e, em alguns casos, podem levar os hospedeiros a óbito (TAYLOR et al., 2017). Nos bubalinos, a infecção é facilitada pelos hábitos desses animais de permanecerem em ambientes favoráveis para o processo de esporulação, com umidade e temperaturas elevadas, o que viabiliza a sobrevivência dos oocistos (DUBEY et al., 2008).

A Eimeriose em búfalos pode levar a perdas econômicas, logo, quando os animais são expostos a altas cargas parasitárias é possível observar redução do ganho de peso ou ainda morte, principalmente de animais jovens (MAMUN et al., 2010). Os sinais clínicos observados em casos de Eimeriose incluem perda de peso, anorexia e diarreia intensa associada a hemorragia (TAYLOR et al., 2017). Porém, nos bubalinos, geralmente a doença é 
assintomática, ou com sinais inespecíficos como pelos arrepiados, baixa conversão alimentar, debilidade e perda de peso (BASTIANETTO et al., 2008).

No estado do Rio Grande do Sul são escassas as informações sobre a infecção por Eimeria spp. em bubalinos, sendo imprescindíveis novos estudos com a finalidade de contribuir para o conhecimento epidemiológico desses parasitos, incentivando a produção de programas de controle sanitário para Eimeriose. Diante do exposto, este trabalho teve como objetivo relatar a ocorrência de Eimeria spp. em bubalinos de aptidão leiteira da microrregião de Camaquã e de Cachoeira do Sul (Rio Grande do Sul, Brasil).

Foram coletadas amostras individuais de fezes diretamente da ampola retal de 539 animais. Sendo 263 animais de uma propriedade rural de Cachoeira do sul, com 202 até um ano de idade e 61 acima de um ano de idade, e 276 animais de uma propriedade rural de Camaquã, com 140 até um ano de idade e 136 acima de um ano de idade. As amostras foram acondicionadas em sacos plásticos e transportadas em caixa isotérmica sob refrigeração. Posteriormente, foram encaminhadas para o Laboratório de Parasitologia do Departamento de Microbiologia e Parasitologia do Instituto de Biologia da Universidade Federal de Pelotas (UFPEL). Os animais foram classificados em dois grupos: jovens (até um ano de idade) e adultos (acima de um ano de idade). A técnica de Gordon e Whitlock modificada (UENO; GONÇALVES, 1998) foi utilizada para obter a contagem de oocistos de Eimeria spp. O resultado foi expresso em oocistos por grama de fezes (OoPG).

Este trabalho foi aprovado pelo Comitê de Ética em Experimentação Animal da Universidade Federal de Pelotas sob o registro no 10606.

A estimativa da ocorrência e o cálculo do intervalo de confiança foram realizados por meio das calculadoras epidemiológicas Epitools (SERGEANT, 2018), com base no método descrito por Reiczigel et al. (2010).

Nas duas propriedades havia animais infectados por Eimeria spp. A ocorrência nos jovens avaliados foi de 41,2\% (IC.95\%: 36\%-46\%) e nos adultos de 16,9\% (IC.95\%: 11,2\%-24,9\%).

Os animais avaliados viviam sob manejo semi-extensivo, apresentavam-se hígidos com raras ocorrências de sinais compatíveis com Eimeriose durante o período de coletas. Tanto as 
fêmeas quanto os bezerros recebiam suplementação no cocho com ração concentrada e silagem. Os bezerros permaneciam junto das mães até atingirem entre oito e 12 meses de idade. Um estudo realizado por Ribeiro et al. (2000), ao avaliar 106 búfalos com idade entre 3 e 45 dias, demonstrou que o gênero Eimeria foi o mais frequentemente identificado, presente em $100 \%$ dos animais com e sem diarreia, com maior prevalência às três semanas de idade. Números semelhantes aos encontrados nos bezerros deste estudo, foram observados nos estados de São Paulo e Paraná, com 45,28\% de positividade em animais apresentando diarreia com até seis meses de idade (GREGORY et al., 2014). Outros autores citam que os sinais clínicos de Eimeriose manifestam-se comumente em animais com altas contagens de oocistos (>10.000 oocistos) (BASTIANETTO et al., 2008), o que raramente ocorreu nas amostras processadas no presente estudo, corroborando com a ausência de sinais clínicos.

Os bezerros foram os animais com ocorrência mais elevada $(41,2 \%)$ do parasito, enquanto os adultos, com mais de um ano de idade, apresentaram uma ocorrência de 16,9\%. Valores semelhantes aos obtidos por Bethencourt et al. (2013) na Venezuela, também em bubalinos leiteiros. A prevalência demonstrou ser menor em animais acima dos 18 meses, o que possivelmente é influenciado pelo desenvolvimento de imunidade após sucessivos contatos dos bubalinos com o protozoário, levando a diminuição na produção de oocistos. Assim, demonstrando que adultos se tornam mais resistentes a Eimeriose pela imunidade adquirida (FAGIOLO et al., 2005; NORONHA et al., 2009).

Baixas contagens de oocistos em bubalinos entre oito e 18 meses e mesmo maiores de 18 meses de idade não apresenta importância clínica, porém os adultos podem demonstrar comportamento de portadores, servindo como uma fonte de infecção permanente para os animais jovens, que ainda não desenvolveram totalmente sua imunidade para os desafios pelo protozoário (LIMA, 2004; MCALLISTER, 2006).

Assim, no presente estudo podemos concluir que Eimeria spp. ocorre em rebanhos bubalinos de aptidão leiteira, acometendo principalmente bezerros. Observou-se que a infecção pelo protozoário, na maioria das vezes, apresentou-se de forma subclínica, podendo, entretanto, interferir no desenvolvimento e rendimento dos animais, afetando 
negativamente a produtividade. Medicações profiláticas e terapêuticas específicas podem ser implementadas, de acordo com a realidade epidemiológica de cada propriedade, para garantir um bom desenvolvimento e a sobrevivência dos animais.

\section{OCCURRENCE OF Eimeria spp. IN DAIRY BUFFALO HERDS IN THE SOUTH OF}

\section{BRAZIL}

\section{ABSTRACT}

$\mathrm{T}$ he creation of buffaloes has been shown to be an important activity in our country, and infections caused by gastrointestinal parasites represent a limiting factor for this species. Eimeria coccidia is one of the primary causes of parasitic diseases in the world, resulting in economic losses and development of clinical signs such as diarrhea, which directly reflects the decrease in production. Considering these facts, the work had as objective to report the occurrence of Eimeria spp. in dairy buffaloes from Camaquã and Cachoeira do Sul microregion, in the state of Rio Grande do Sul, Brazil. A total of 539 faecal samples were collected directly from the rectal ampule of buffaloes of the two dairy farms, which were divided into two categories: young ( $<1$ year old) and adults ( $>1$ year old); samples were processed by the modified Gordon and Whitlock technique, which was used to obtain the oocysts count of Eimeria spp. and this result was expressed in oocysts per gram of faeces (OoPG). The apparent prevalence was calculated, where it was observed that $41.2 \%$ of the young buffaloes were positive for Eimeria spp. and only $16.9 \%$ of adults were positive. For a good control and effective measures to combat eimeirosis in the buffalo herds of Rio Grande do Sul, it is necessary to know the occurrence of this protozoan in buffaloes.

Keywords: Parasites. Protozoa. Coprological examination. Bubalus bubalis. 


\section{OCURRENCIA DE Eimeria spp. EN LOS REBAÑOS DE BUFALINOS LECHEROS EN \\ EL SUR DE BRASIL}

\section{RESUMEN}

a creación de bufalinos es una actividad importante en la actividad pecuaria en Brasil, y las infecciones por parásitos gastrointestinales representan un punto limitante para esta especie. El coccidio del género Eimeria es una de las causas principales de enfermedades parasitarias de presencia mundial, causando pérdidas económicas y desarrollo de signos clínicos como la diarrea, lo que refleja directamente una disminución en la producción. Ante estos hechos, el presente estudio tuvo como objetivo describir la presencia de Eimeria spp. en bufalinos productores de leche de las microrregiones de Camaquã y Cachoeira do Sul, Rio Grande do Sul, Brasil. Se obtuvieron 539 muestras de heces, directamente de la ampolla rectal. Los búfalos se dividieron en dos categorías: jóvenes (menores de un año) y adultos (mayores de un año). Las muestras fueron procesadas por la técnica de Gordon y Whitlock modificada, para obtener el recuento de ooquistes de Eimeria spp. Este resultado se expresa en ooquistes por gramo de heces (OoPG). Como resultado, se encontró que el $41,2 \%$ de los búfalos jóvenes y el 16,9\% de los adultos eran positivos para Eimeria spp. Este estudio demuestra la presencia de Eimeria spp. parasitando a los búfalos de las microrregiones de Camaquã y Cachoeira do Sul, afectando principalmente a los animales jóvenes.

Palabras clave: Parásitos. Protozoarios. Exámenes coprológicos. Bubalus bubalis.

\section{REFERÊNCIAS}

BASTIANETTO, E; FREITAS, C. M. V.; BELLO, A. C. P. P.; et al. Primeiro diagnóstico de Eimeria bareillyi (Apicomplexa: Eimeridae) nas fezes de bezerros bubalinos (Bubalus bubalis) naturalmente infectados no Estado de Minas Gerais, Brasil. Revista Brasileira de Parasitologia Veterinária, v. 17, n. 1, p. 234-238, 2008.

BERNARDES, O. Bubalinocultura no Brasil: situação e importância econômica. Revista Brasileira de Reprodução Animal, v. 31, n. 3, p. 293-298, 2007.

BETHENCOURT, A. M.; QUIJADA, J. J.; CABRERA, P.; et al. Prevalencia y abundancia de huevos de estróngilos digestivos y ooquistes. Revista de La Facultad de Ciencias Veterinarias, v. 54, n. 1, p. 17-28, 2013.

DANTAS, P. C. S.; LIMA, D. S.; OLIVEIRA, F. J.; et al. Ocorrência de parasitos gastrintestinais em vacas leiterias e respectivos bezerros durante o período de amamentação, na fazenda São Paulino, Município de Itapicuru/BA. Scientia Plena, v. 11, n. 4, p. 1-7, 2015. 
DUBEY, J. P.; WOUDA, W.; MUSKENS, J. Fatal intestinal coccidiosis in a three-week-old buffalo calf (Bubalus bubalus). Journal of Parasitology, v. 94, n. 6, p. 1289-1294, 2008.

FAGIOLO, A.; RONCORONI, C.; LAI, O.; BORGHESE, A. Buffalo pathologies. In: BORGHESE, A. Buffalo Production and Research. Roma: FAO, 2005. P. 249-296.

GREGORY, L.; ROSSI, R. S.; MENDES, J. P. G.; et al. Occurrence of the main bacterial and parasite agents on diarrhetic feces of buffalo calves in the states of São Paulo and Paraná. Arquivos do Instituto Biológico, v. 81, n. 2, p. 180-185, 2014.

IBGE - INSTITUTO BRASILEIRO DE GEOGRAFIA E ESTATÍSTICA. Censo Agropecuário 2006. Disponível em: <https://sidra.ibge.gov.br/tabela/281\#resultado> .

IBGE - INSTITUTO BRASILEIRO DE GEOGRAFIA E ESTATÍSTICA. Censo Agropecuário 2017, tabelas, pecuária, efetivo da pecuária. Disponível em:

$<$ https://www.ibge.gov.br/estatisticas/economicas/agricultura-e-pecuaria/21814-2017censo-agropecuario.html?=\&t=resultados $>$.

LIMA, J. D. Coccidioses dos ruminantes domésticos. Revista Brasileira de Parasitologia Veterinária, v. 13, supl. 1, p. 9-13, 2004.

MAMUN, M. A. A.; BEGUM, N.; SHAHADAT, H. M.; et al. Ectoparasites of buffaloes (Bubalus bubalis) in Kurigram district of Bangladesh. Journal of Bangladesh Agricultural University, $v$. 8, n. 1, p. 61-66, 2010.

MCALLISTER, M. M. Protozoosis of the Calf: Giardia, Cryptosporidium, Eimeria, Sarcocystis, Neospora. In: WORLD BUIATRICS CONGRESS, 24, 2006, Nice. PROCEEDINGS. Nice: WBC, 2006. Disponível em:

http://citeseerx.ist.psu.edu/viewdoc/download?doi=10.1.1.527.9829\&rep=rep1\&type=pdf

NORONHA, A. C. F.; STARKE-BUZETTI, W. A.; DUSZYNSKY, D. W. Eimeria spp. in Brazilian water buffalo. Journal of Parasitology, v. 95, n. 1, p. 231-234, 2009.

REICZIGEL, J.; FÖLDI, J.; OZSVÁRI, L. Exact confidence limits for prevalence of a disease with an imperfect diagnostic test. Epidemiology and Infection, v. 138, n. 11, p. 1674-1678, 2010.

RIBEIRO, M. G.; LANGONF, H.; JEREZ, J. A.; et al. Indentification of enteropathogens from buffalo calves with and without diarrhoea in the Ribeira Valley, State of São Paulo, Brazil. Brazilian Journal of Veterinary Research and Animal Science, v. 37, n. 2, p. 159-165, 2000.

SERGEANT, E. S. G. Epitools epidemiological calculators. Ausvet Pty Ltd. 2018. Disponível em: <http://epitools.ausvet.com.au>. 
TAYLOR, M. A.; COOP, R. L.; WALL, R. L. Parasitologia Veterinária. 4. ed. Rio de Janeiro: Guanabara Koogan, 2017. 1052p.

UENO, H. GONÇALVES, P. C. Manual para diagnóstico das helmintoses de ruminantes. 4. ed. Porto Alegre: JICA, 1998. 72p.

VILELA, V. L. R.; FEITOSA, T. F.; BRASIL, A. W. L.; et al. Prevalence of gastrointestinal parasites in buffaloes in the state of Paraíba and the first report of Cystoisospora spp. in buffaloes in Brazil. ARS Veterinária, v. 33, n. 1, p. 26-30, 2017.

Autor para correspondência: Sara Patron Motta. Laboratório de Parasitologia, Instituto de Biologia, UFPEL, Campus Universitário s/n, Capão do Leão (RS). sarapatron@hotmail.com 